\title{
Health impact assessment of air pollution: providing further evidence for public health action
}

\author{
M. Martuzzi*, M. Krzyzanowski ${ }^{\#}$, R. Bertollini*
}

Health impact assessment of air pollution: providing further evidence for public health action. M. Martuzzi, M. Krzyzanowski, R. Bertollini. C ERS Journals Ltd 2003.

ABSTRACT: Many epidemiological studies have demonstrated the importance of air pollution as a risk factor and characterised dose-response relationships between health endpoints and pollutants.

The association between particulate matter (PM) and health is generally regarded as causal, and a nonthreshold linear relationship with, for example, mortality and hospital admission has been observed in several settings.

The ubiquitous PM air pollution is likely to have a large overall impact on human health, even if risks are relatively small. There have recently been a large number of papers reporting quantitative estimations of the health impact of $\mathrm{PM}$ on health, as measured by the proportion of excess events that are attributable to PM exposures in the general population, mainly in industrialised countries. For example, in the eight largest Italian cities it has been estimated that concentrations beyond $30 \mu \mathrm{g} \cdot \mathrm{m}^{-3}$ are responsible for about 3,500 extra deaths per year. A similar study has been carried out for France, Austria and Switzerland.

These evaluations fill a knowledge gap between the laboratory and clinical studies on the pathophysiological mechanisms, the epidemiological research on the nature and strength of the association at the population level, and the risk management needs for developing appropriate preventive policies.

Some limitations in the methodology deserve further research, however health impact assessment type studies are informative and effective tools of communication with the general public and policy makers.

Eur Respir J 2003; 21: Suppl. 40, 86s-91s.
*Rome Office, Rome, Italy, ${ }^{\#}$ Bonn Office of the World Health Organization - European Centre for Environment and Health.

Correspondence: M. Martuzzi

Rome Office

Via F Crispi 10

00187 Rome

Italy

Fax: 39064877599

E-mail: mam@who.it

Keywords: Air pollution elderly

health impact assessment public health

Received and accepted: April 122002
The scientific evidence on the health effects of ambient air pollution has been growing in recent years. Several questions remain open, but many epidemiological studies have demonstrated the importance of air pollution as a risk factor for mortality and morbidity [1-9]. For several specific health endpoints and pollutants, the associations have been quantified and concentration-response relationships have been characterised. Among these pollutants, the role of suspended particulate matter (PM) has been especially investigated with regard to its short- and long-term effects on mortality and morbidity. Most of the scientific evidence available to date concerns PM10 and PM2.5 (particles with a 50\% cut-off aerodynamic diameter of $<10$ and $<2.5 \mu \mathrm{m}$, respectively), although the role of finer particles is of increasing interest. This evidence has prompted the World Health Organization (WHO) to include air pollution and its health effects in its agenda [10-12].

Although the biological mechanism of action is not yet fully understood, the association between PM and health is generally regarded as causal [13], and a nonthreshold concentrationresponse relationship with, for example, mortality and hospital admission has been observed in several settings. Cohort studies conducted in the USA found increase in total and cardio-respiratory mortality in populations of cities with higher long-term mean PM10 concentrations [14-16]. Shortterm studies have demonstrated effects occurring shortly after elevated concentration days: hospital admission [17-21], incidence of new cases of bronchitis and occurrence of respiratory symptoms and asthma exacerbation [22-24], for adults and children. Increase in daily mortality following days of elevated
PM concentrations has also been described by many studies worldwide $[25,26]$. With the possible exception of acute bronchitis in children, the relative risks for all these adverse outcomes are of the order of a few per cent units increase for each $10 \mu \mathrm{g} \cdot \mathrm{m}^{-3}$ increase in PM10 concentration. Compared to other risk factors in epidemiology, these effects are relatively small, but, on the other hand, due to the ubiquitous nature of PM air pollution, virtually everyone in industrialised societies is exposed, especially urban populations. So there are concerns that the overall impact on human health can be large, and the associated social and economic costs can be high.

The consistency of the findings of epidemiological studies and the availability of reliable concentration-response models, together with the availability of extensive concentration data routinely collected by monitoring stations, have recently enabled the quantitative estimation of the health impact of PM on the health of populations of several countries in the world. The use of epidemiological information for health impact assessment in general has been recently evaluated [27], and specific criteria for using air quality monitoring data for health impact assessment have been described [28]. In evaluations of air pollution health impact, PM has often been used as a summary indicator of air quality, and the findings describe the burden imposed on health by PM pollution (mostly in urban settings), and in some cases the associated economic costs, under the assumption that PM might be responsible for direct effects but also capture the effects of other correlated pollutants. The cost-benefit question that underlies these evaluations is "what are the health gains 
potentially obtainable adopting policies of abatement of emissions of PM and other pollutants?", a piece of information that politicians and community members increasingly require to inform the decision-making process. A WHO working group has recently addressed the question of defining appropriate methods for air pollution health impact assessment [29].

The published analyses have indicated that ambient PM exposure at concentrations normally measured in industrialised countries, especially in urban areas, result in large numbers of excess adverse health events [30-35]. Generally speaking, the health burden, or impact, is measured by the number of adverse health events that are due to PM exposures in the population under study.

The general approach that has been followed is based on these steps: 1) establish a set of health endpoints that are known to be associated to PM exposure and for each of these do the following; 2) identify a concentration-response coefficient and its confidence interval (CI), using data published in the epidemiological literature; 3) estimate the proportion of the events observed in the study population that is attributable to PM concentration, and the corresponding absolute number of cases over a given time period; this is a function of the concentration-response coefficients, the measured PM10 concentrations, and the observed incidence rates or prevalences of the health endpoints in the study population. The overall sum of these can lead to economic evaluations, that are not further discussed in this paper. These three steps are described in the following three sections.

\section{Choice of health endpoints}

Published PM health impact assessment studies [30-35] have mainly addressed mortality in people $>30$ yrs and excluding accidental causes, hospital admissions for respiratory and cardiovascular disease, incidence of new cases of chronic bronchitis, occurrence of respiratory symptoms and asthma exacerbation episodes, separately for adults and children (table 1). This choice reflects the need to include endpoints for which evidence from epidemiological studies is reliable and based on concentration-response coefficients. It also indicates the underlying cost-benefit perspective, i.e. estimates are not only intended as "impact" on health, but ideally as "gains" or benefits that would be achieved by reducing average concentrations (to be weighed against the costs). Thus, changes in acute mortality, which are very consistently associated with PM short-term fluctuations, are

Table 1.-Health endpoints used for particulate matter health impact assessment

\begin{tabular}{lc}
\hline Cause & $\begin{array}{c}\text { Relative risk per } \\
10 \mu \mathrm{g} \cdot \mathrm{m}^{-3} \mathrm{PM} 10 \\
(95 \% \mathrm{CI})\end{array}$ \\
\hline $\begin{array}{l}\text { Mortality (adults aged } \geqslant 30, \\
\quad \text { excluding accidental causes) }\end{array}$ & $1.043(1.026-1.061)$ \\
Hospital admissions for CVD causes & $1.009(1.006-1.013)$ \\
Hospital admissions for respiratory & $1.016(1.013-1.020)$ \\
$\quad$ disease & $1.306(1.135-1.502)$ \\
Acute bronchitis (aged $<15)$ & $1.051(1.047-1.055)$ \\
Asthma exacerbation $($ aged $<15)$ & $1.004(1.0-1.008)$ \\
Asthma exacerbation (aged $\geqslant 15)$ & $1.094(1.079-1.109)$ \\
Restricted activity days (aged $\geqslant 20)$ & $1.07(1.02-1.11)$ \\
Occurrence of respiratory symptoms &
\end{tabular}

PM10: particles with a 50\% cut-off aerodynamic diameter of $10 \mu \mathrm{m}$; CVD: cardiovascular disease; CI: confidence interval. not included, as there are some doubts that reduction in daily peak concentrations would result, over a long period, in gains as predicted by the observed daily associations [36-38]. Similarly other physiological endpoints, seldom measured at the population level, such as pulmonary function, are not normally included in the impact estimates.

\section{Concentration-response coefficients}

Once the relevant health outcomes have been identified, a summary concentration-response coefficient must be derived from the epidemiological literature. For example, most authors use estimates of effects of long-term exposure on mortality from POPE et al. [15] and DOCKERY et al. [14]. When many studies provide information on exposure-response association, a meta-analysis of their results is conducted to derive a common estimate. Typically, the study with less uncertainty carries more weight in the meta-analysis and in deriving the final combined estimate. This procedure applied to the results of the cohort studies on mortality, produces a relative risk of $1.043(95 \%$ CI $1.026-1.061)$ for $10 \mu \mathrm{g} \cdot \mathrm{m}^{-3}$ of long-term average PM10 based on log-linear (i.e. exponential) Poisson regression models. Given the limited numerical magnitude of the effects, the relationship can be taken to be linear (fig. 1) since the difference between the two models is small. More important bias may arise from extrapolating the association beyond the exposure levels observed in the studies providing the evidence.

\section{Estimating the impact}

The impact is estimated separately for each health outcome. The natural metric for measuring a health impact is the portion of the observed occurrence of an outcome that is attributable to PM. This can be expressed in terms of proportions or percentages or absolute number of cases. The latter seems to be the favourite of both scientists and nonspecialised people, perhaps because of its clarity and ease of communication. To work out attributable risks, one needs to know, besides the concentration-response coefficient, the distribution of exposure in the population, which in the case of

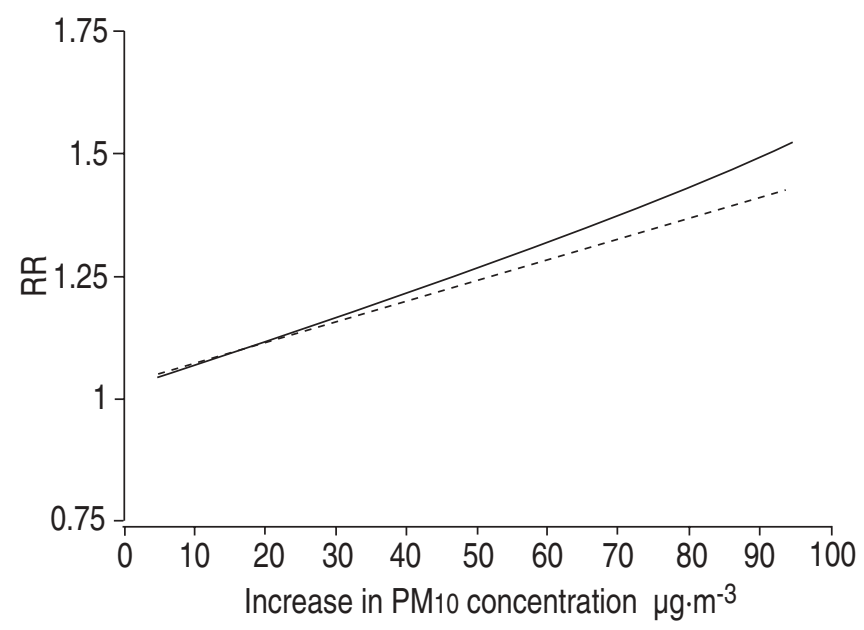

Fig. 1.-Different estimates of relative risk (RR) of mortality associated with PM10, based on different Poisson regression models ${ }^{\#}$. - : log linear; ----: linear. " ${ }^{*}$ Derived from RR 1.043 (95\% confidence interval 1.026-1.061), assuming that an increase from 0 to $20 \mu \mathrm{g} \cdot \mathrm{m}^{-3}$, for example, results in $\mathrm{RR}=1+(.043 \times 2)=1.086$ (linear) versus $1.043^{2}=1.088$ (log-linear). 
ambient PM pollution, consists of the average concentration measured in the city or area where the population live. Typically, available monitoring data allows characterisation of the concentration that applies to large groups of people (all residents in a city or a neighbourhood), and everyone in such groups is assumed to be exposed to the same PM concentration (implications discussed below). With this information it is possible to calculate the attributable risk as a proportion, while to obtain the absolute number of attributable cases it is necessary to know, in addition, the observed rates of disease or mortality occurrence in the population under study.

There has been some discussion in the literature about the meaning and the interpretation of attributable risks. There are conflicting view-points and some paradoxical situations have been pointed out [39]. For the purposes of the current study it seems satisfactory to think of attributable risk as the proportion of disease occurrence that would be prevented if the rates observed in the unexposed group prevailed in the whole population. This is a rather cautious definition, far less binding than the proportion of disease occurrence that would be prevented if exposure was removed.

The two definitions are equivalent only under certain circumstances. Deriving a meaningful attributable risk from a dose-response relationship and the distribution of exposure in the population assumes full causality of the observed association. Interpreting attributable risks as a measure of potential benefits following abatement policies, additionally requires reversibility of the exposure-disease process, which raises the question "what is the nature of the causal link?" (which is not fully known for PM and health). A possible reason why attributable risks may not be fully reflected by gains from public health actions is that the exposure-disease association might take place through some intermediate steps, and removal of one factor in the chain might result only in limited reduction of the following factor. Also, attributable risk estimates are based on the hypothetical situation where only the exposure of interest is removed, and all other determinants are unchanged (this also explains why attributable risks for different factors add up to $>100 \%$ ), which is difficult to imagine in reality. If none of these problems occur, then the two definitions of attributable risk above are equivalent.

Since the question asked by policy makers is "how many cases would be prevented if concentrations were reduced to $\mathrm{X}$ ?", the cautious answer would therefore be "Y fewer cases would be observed, 'if' the association observed in epidemiological studies is entirely causal and 'if' and 'when' rates observed in the unexposed can be achieved following exposure reduction".

In fact, another important factor is the temporal scale of effects, i.e. the latency times from exposure to adverse event. For acute effects, this is not too problematic, as they follow exposure by a few days, but in the case of long-term exposure on mortality, this is uncertain. For both short- and long-term effects, adaptive responses and the role of competing risk factors might break the identity between health impact (estimated retrospectively) and potential gain (estimated prospectively), but for long-term exposures and mortality accumulated over a long time period the additional question arises as to how long before a reduction in PM concentration is followed by a reduction in mortality. In addition, an "attributable death" is inevitably an anticipated death, so the most relevant public health measure is how long is the displacement time, i.e. how many years or months of life are lost. These considerations led some authors to estimate PM impact on mortality not in terms of attributable proportions or number of cases, but in terms of person-time lost, expressed as reduction in life expectancy, and to describe how gains would take place under different hypotheses on latency time [33]. It was estimated, for example, that for people alive at the beginning of 2000 in England and Wales, assuming a $10-\mathrm{yr}$ latency period, a reduction of $10 \mu \mathrm{g} \cdot \mathrm{m}^{-3}$ of PM10 would result in a gain of 15.17 million life years, i.e. an increase of a few months life expectancy for the whole population, achieved $10 \mathrm{yrs}$ after the concentrations are reduced.

\section{Analytical methods}

The algebra for estimating attributable risks and attributable number of cases, as a function of concentrationresponse coefficients, average concentrations and observed prevalences, is simple. The rationale is that the observed prevailing rate is the rate in the population exposed to the current measured concentrations. Using the coefficient, one calculates the rate that would prevail if the population was unexposed, and the comparison between this predicted rate and the observed rate provides the attributable risk and the attributable number of cases. For obtaining CIs, the estimation is repeated using, in place of the concentrationresponse coefficient, its upper and lower confidence limits. This process raises an important question: what is meant by "unexposed"? In principle it is possible to use, as reference level for exposure, the zero concentration, but this has not been done in published studies, as it is not useful to estimate the total health impact of all PM, because it is not realistic to envisage zero concentrations. KUENZLI et al. [30] for example, used $7.5 \mu \mathrm{g} \cdot \mathrm{m}^{-3}$ as reference for PM10, as this is regarded as the natural background concentration; results are then to be interpreted as health impact of man-made PM10. Other authors give estimates of attributable risks using different reference levels (e.g. 20 and $30 \mu \mathrm{g} \cdot \mathrm{m}^{-3}$ ) [34], or associated to changes in PM10 concentration of $5,10,15$ and $25 \mu \mathrm{g} \cdot \mathrm{m}^{-3}$ [33], in order to illustrate the potential benefits associated with different reduction policy scenarios. An advantage of the linear approximation is that it is possible to express attributable risk per $10 \mu \mathrm{g} \cdot \mathrm{m}^{-3}$, without specifying a fixed reference level, as was done by KUENZLI et al. [30]. This allows the reader to work out the attributable risks and potential gains at any reference value; on the other hand gains per unit PM changes might cause some confusion as they can be more easily mistaken for constant values, which can be extrapolated across studies, whereas they strictly apply to the population under study.

The algorithm for calculating in practice the attributable number of cases, E, given a health outcome is reported in figure 2 .

\section{Interpretation of health impact assessments}

The approach outlined above has been adopted by most published studies aimed at assessing the health impact of PM. As discussed, while deriving the estimates may be simple, their implications are not straightforward. There are several limitations that should be considered. As in many studies in the environmental field, one of the most important sources of error is poor exposure assessment. The impact studies rely on measures of PM concentration that are averaged over long time periods and across large populations, such as all residents of large cities. Obviously these mean values result from a distribution of concentrations that might have different degrees of variability, even of large magnitude, and more importantly they approximate roughly the relevant unknown individual exposure. Although this is a potentially serious limitation, it must be said that impact studies are consistent with the epidemiological studies which provide the relevant 


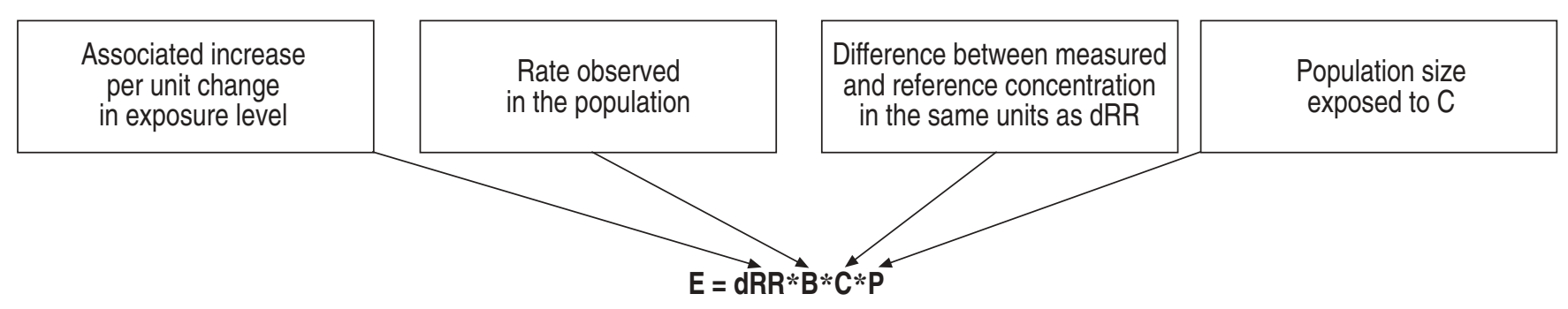

Fig. 2. - Algorithm for calculating the attributable number of cases (E) of a health outcome.

information: the concentration-response coefficients are estimated in epidemiological studies using average measures of concentration, and the same measures are used for impact assessment. In addition, it has been suggested that, assuming the existence of an appropriate individual measure of exposure (which has not yet been identified), average concentrations are an approximation which involves Berkson type error and risk estimates are therefore unbiased [40]. However, while this consistency is appealing, it has not yet been demonstrated clearly that impact estimates are unbiased; perhaps even more importantly, it is not clear whether estimates of uncertainty, calculated as a function of only the concentration-response coefficient, are correct, or if other sources of error should be included.

A second important question concerning the interpretation is the validity of extrapolating results from epidemiological studies carried out on a population to other populations for impact assessment. While substantial differences in terms of susceptibility to air pollution seem unlikely, there are other factors that may produce bias, such as: differences in daily pattern of activity, climatic conditions, housing, etc. that would result in different exposures from the same ambient concentration; different importance of confounding factors that might not have been properly controlled for in the epidemiological studies; different techniques in air pollution concentration measurement, and others. In principle it would be preferable to use coefficients estimated from the same population for which the impact assessment was done, however this is rarely possible. For long-term mortality, for example, large and costly population-based cohort studies would be needed.

A third limitation is in the use of PM as an indicator for the mix of pollutants to which people are exposed. Undoubtedly PM10 has been measured reliably in many parts of the world, especially in urban areas, and it is correlated with many other gaseous pollutants. In addition it would be more difficult to estimate the health impact of other pollutants, as quantitative dose-response information is more limited than for PM10. However, even if the estimation was done, it would not be correct to sum up the impact estimates to obtain an overall air pollution impact, except if different health outcomes were considered. This applies to most common pollutants in the urban setting (PM, nitrogen oxides, sulphur dioxide, carbon monoxide), with the exception of ozone $\left(\mathrm{O}_{3}\right)$, which is uncorrelated with the other pollutants. $\mathrm{O}_{3}$ was included in a recent impact assessment for four large American cities [31].

Finally, it is important to consider the question of the susceptible subgroups of the population. It is possible that the associations observed in the epidemiological studies are driven by selected subsets of the population, defined by age, profession, social class or physiological parameters. If this is the case, it would be another factor limiting the validity of extrapolating risks from a population to another if the proportions of the sensitive groups differ between populations.

These limitations have been acknowledged by all authors of
PM impact studies, which are based on a common rationale and, although there are some differences, share most of the methodology. It has been suggested that this methodology produces estimates of at least part of the overall air pollution health impact [30], for example by considering PM only and ignoring other pollutants' effects, and by not considering all relevant health outcomes. Thus, these impact estimates are considered to underestimate the overall health impact, and therefore be "prudent" in terms of developing and adopting abatement policies without incurring excessive societal costs.

\section{Discussion}

Available impact studies indicate that the burden on health in populations of industrialised countries is large. For example, recent studies carried out in Europe have evaluated the health impact of PM10, estimating that ordinary concentrations account for tens of thousands of deaths per year in France, Switzerland and Austria [30], thousands of deaths per year in the eight largest Italian cities [34], millions of person-years in England and Wales, and hundreds of thousands of personyears in the Stockholm County (based on PM2.5 concentrations) [32]. Region-wide analysis conducted by WHO is consistent with those individual studies [35]. Mortality tends to attract most of the attention, but the attributable morbidity and hospitalisation is also very large.

These kinds of evaluations fill a knowledge gap between the laboratory and clinical studies on the pathophysiological mechanisms, the epidemiological research on the nature and strength of the association at the population level, and risk management. These risk assessment studies are necessary steps preceeding the development of appropriate preventive policies. The limitations discussed above should be taken into consideration in order to make these estimates more reliable, and make a stronger case for public health action.

Further work, in particular, seems to be needed to develop second generation impact assessment studies, with regard to several methodological aspects, illustrated in table 2. With regard to a clearer understanding of the impact estimates for hospital admissions, it is important to point out that they might be subject to a harvesting effect as well as mortality [41]. Other aspects which require consideration include: the link between "attributable" and "preventable" risks, and the temporal dimension; consideration of susceptible subgroups such as children, the elderly, people with respiratory conditions or allergies; and the inclusion of these impact assessments into integrated impact assessments of, for example, transport policies, where a variety of other direct and indirect effects are taken into consideration.

Furthermore, there is an urgent need for improvement of the databases for health impact assessment in developing countries. Scarce air quality data indicate that air pollution may cause significant burden to health of their residents. However the lack of locally conducted epidemiological studies 
Table 2. - Further work needed to develop second generation impact assessment studies

Validity of using large population average concentrations

Validity of extrapolating risk estimates across populations

Reliability of uncertainty estimates

Treatment of multiple pollutants

Appropriateness of the linear concentration-response model for all health outcomes

Inclusion of further health outcomes

Clearer understanding of the impact estimates for hospital admissions, which might be subject to a harvesting effect as well as mortality [28]

Link between "attributable" and "preventable" risks, and the temporal dimension

Consideration of susceptible subgroups such as children, the elderly, people with respiratory conditions or allergies

Inclusion of these impact assessment into integrated impact assessments of, for example, transport policies, where a variety of other direct and indirect effects are taken into consideration

raises questions about the applicability of extrapolations based on the USA and European investigations.

Notwithstanding the limitations and the need to develop more appropriate methods, health impact assessment studies have proven informative and effective as a means of communication with the general public, decision makers and other interested parties, to feed available scientific evidence into the policy making process, and to draw attention to the urgent need to take action to reduce an important risk factor for the health of millions of people worldwide.

\section{References}

1. Krzyzanowski M, Wojtyniak B. Ten-year mortality in a sample of an adult population in relation to air pollution. J Epidemiol Community Health 1982; 36: 262-268.

2. Jedrychowski W, Krzyzanowski M, Wojtyniak B. Confronting the effects of smoking and air quality on the development of chronic respiratory diseases. Tokai J Exp Clin Med 1985; 10: 323-330.

3. Quackenboss JJ, Krzyzanowski M, Lebowitz MD. Exposure assessment approaches to evaluate respiratory health effects of particulate matter and nitrogen dioxide. J Expo Annal Environ Epidemiol 1991; 1: 83-107.

4. Krzyzanowski M, Quackenboss JJ, Lebowitz MD. Chronic respiratory effects of indoor formaldehyde exposure. Environ Res 1990; 52: 117-125.

5. Krzyzanowski M, Wojtyniak B. Air pollution and daily mortality in Cracow. Public Health Rev 1991-92; 19: 73-81.

6. Di Paola M, Mastrantonio M, Comba P, Grignoli M, Maiozzi P, Martuzzi M. Territorial distribution of mortality from malignant tumors of the pleura in Italy. Ann Ist Super Sanita 1992; 28: 589-600.

7. Comba P, Di Paola M, Martuzzi M, Pirastu R. Asbestosrelated mortality in Italy: a geographical approach. Med Lav 1997; 88: 293-301.

8. Martuzzi M, Comba P, De Santis M, et al. Asbestos-related lung cancer mortality in Piedmont, Italy. Am J Ind Med 1998; 33: 565-570.

9. Brunekreef B, Dockery DW, Krzyzanowski M. Epidemiologic studies on short-term effects of low levels of major ambient air pollution components. Environ Health Perspect 1995; 103: Suppl. 2, 3-13.

10. Bertollini R, Dora C, Krzyzanowski M, Stanners D. Environment and health 1, overview and main European issues. WHO Reg Publ Eur Ser 1996; 68: 4-56.

11. van der Heijden KA, Krzyzanowski M. Priorities in environmental health risks related to air pollution throughout Europe. Zentralbl Bakteriol 1994; 281: 296-302.

12. Krzyzanowski M. Methods for assessing the extent of exposure and effects of air pollution. Occup Environ Med 1997; 54: 145-151.

13. World Health Organization. Air Quality Guidelines for Europe. WHO Regional Office for Europe. WHO Regional
Publications, European Series. No. 91. World Health Organization, Copenhagen, 2000.

14. Dockery DW, Pope AC, III, Xu X, et al. An association between air pollution and mortality in six U.S. cities. $N$ Engl J Med 1993; 329: 1753-1759.

15. Pope CA, III, Thun MJ, Namboodiri MM, et al. Particulate air pollution as a predictor of mortality in a prospective study of U.S. adults. Am J Respir Crit Care Med 1995; 151: 669-674.

16. Abbey DE, Nishino N, McDonnell WF, et al. Long-term inhalable particles and other air pollutants related to mortality in nonsmokers. Am J Respir Crit Care Med 1999; 159: 373-382.

17. Poloniecki JD, Atkinson RW, de Leon AP, Anderson HR. Daily time series for cardiovascular hospital admissions and previous day's air pollution in London, UK. Occup Environ Med 1997; 54: 535-540.

18. Wordley J, Walters S, Ayres JG. Short term variations in hospital admissions and mortality and particulate air pollution. Occup Environ Med 1997; 54: 108-116.

19. Anderson HR, Ponce DL, Bland JM, Bower JS, Emberlin J, Strachan DP. Air pollution, pollens, and daily admissions for asthma in London 1987-92. Thorax 1998; 53: 842-848.

20. Schwartz J. PM10, ozone, and hospital admissions for the elderly in Minneapolis-St. Paul, Minnesota. Arch Environ Health 1994; 49: 366-374.

21. Schwartz J, Slater D, Larson TV, Pierson WE, Koenig JQ. Particulate air pollution and hospital emergency room visits for asthma in Seattle. Am Rev Respir Dis 1993; 147: 826-831.

22. Dockery DW, Pope CA, III. Acute respiratory effects of particulate air pollution. Annu Rev Public Health 1994; 15: 107-132.

23. Braun-Fahrlander C, Vuille JC, Sennhauser FH, et al. Respiratory health and long-term exposure to air pollutants in Swiss schoolchildren. SCARPOL Team. Swiss Study on Childhood Allergy and Respiratory Symptoms with Respect to Air Pollution, Climate and Pollen. Am J Respir Crit Care Med 1997; 155: 1042-1049.

24. Forastiere F, Corbo GM, Pistelli R, et al. Bronchial responsiveness in children living in areas with different air pollution levels. Arch Environ Health 1994; 49: 111-118.

25. Katsouyanni K, Touloumi G, Spix C, et al. Short-term effects of ambient sulphur dioxide and particulate matter on mortality in 12 European cities: results from time series data from the APHEA project. Air Pollution and Health: a European Approach. BMJ 1997; 314: 1658-1663.

26. Samet JM, Zeger S, Dominici F, . The National Morbidity, Mortality, and Air Pollution Study. Part II: Morbidity, Mortality, and Air Pollution in the United States. 94, Part II. Cambridge, MA, Health Effects Institute, 2000.

27. World Health Organization. Evaluation and Use of Epidemiological Evidence for Environmental Health Risk Assessment. WHO Regional Office for Europe. Guideline Document. Copenhagen, World Health Organization, 2000.

28. World Health Organization. Monitoring Ambient Air Quality for Health Impact Assessment. WHO Regional Office for Europe. WHO Regional Publications, European 
Series. No. 85. Copenhagen, World Health Organization, 1999.

29. World Health Organization. Quantification of the Health Effects of Exposure to Air Pollution. Copenhagen, World Health Organization, Regional Office for Europe, 2001.

30. Kuenzli N, Kaiser R, Medina S, et al. Public-health impact of outdoor and traffic-related air pollution: a European assessment. Lancet 2000; 356: 795-801.

31. Cifuentes L, Borja-Aburto VH, Gouveia N, Thurston G, Davis DJ. Assessing the health benefits of urban air pollution reductions associated with climate change mitigation (2000 2020): Santiago, Sao Paulo, Mexico City, and New York City. Environ Health Perspect 2001; 109: Suppl. 3, 419425.

32. Bellander T, Svartengren M, Berglind N, Staxler L, Jarup L. SHAPE - the Stockholm study on health effects of air pollution and their economic consequences. Part II: particulate matter, nitrogen dioxide, and health effects, exposureresponse relations and health consequences in Stockholm county. Publication no. 160. Stockholm, Vagverket, 1999.

33. Hurley JF, Holland MR, Markandia A, et al. Towards assessing and costing the health impacts of ambient particulate air pollution in the UK. Edinburgh, Institute of Occupational Medicine, 2000.

34. World Health Organization. Health impact assessment of air pollution in the eight major Italian cities. http://www.euro.
who.int/document/E75492.pdf. Date accessed: September 25 2002.

35. Overview of the Environment and Health in Europe in the 1990s. Background Document, 3rd Ministerial Conference on Environment and Health, London 16-18 June 1999. World Health Organization, Regional Office for Europe, Copenhagen, 1999 (EUR/ICP/EHCO 0202 05).

36. McMichael AJ, Anderson HR, Brunekreef B, Cohen AJ. Inappropriate use of daily mortality analyses to estimate longer-term mortality effects of air pollution. Int $J$ Epidemiol 1998; 27: 450-453.

37. Kunzli N, Medina S, Kaiser R, Quenel P, Horak F Jr, Studnicka M. Assessment of deaths attributable to air pollution: should we use risk estimates based on time series or on cohort studies? Am J Epidemiol 2001; 153: 1050-1055.

38. Martuzzi M. Re: "Assessment of deaths attributable to air pollution: should we use risk estimates based on time series or on cohort studies?" Am J Epidemiol 2001; 154: 974-975.

39. Greenland S, Robins JM. Conceptual problems in the definition and interpretation of attributable fractions. $\mathrm{Am} \mathrm{J}$ Epidemiol 1988; 128: 1185-1197.

40. Thomas D, Stram D, Dwyer J. Exposure measurement error: influence on exposure-disease. Relationships and methods of correction. Annu Rev Public Health 1993; 14: 69-93.

41. Bellander T. Public health and air pollution. Lancet 2001; 357: 69-70. 